\title{
Third sound measurements as capillary condensation probe in porous materials
}

\author{
J. P. Laheurte, J. C. Noiray and J. P. Romagnan \\ Laboratoire de Physique de la Matière Condensée, Université de Nice, 06034 Nice Cedex, France
}

(Reçu le 19 juillet 1979, accepté le 16 octobre 1979)

\begin{abstract}
Résumé. - Nous présentons des mesures de troisième son et des isothermes d'adsorption dans des films d'He ${ }^{4}$ permettant de détecter la condensation capillaire dans des matériaux poreux tels que poudre d'alumine et graphite exfolié comprimé. Le troisième son se révèle une technique extrêmement utile pour observer de tels effets.
\end{abstract}

\begin{abstract}
We present third sound measurements coupled with adsorption isotherms in $\mathrm{He}^{4}$ film which show clearly capillary condensation in porous materials such as compressed exfoliated graphite and alumina powder. Third sound appears to be a very useful tool for detecting such effects.
\end{abstract}

Recent measurements of sound velocity in superfluid helium adsorbed on alumina powder [1] and adsorption isotherms of $\mathrm{He}^{4}$ with a large variety of substrates [2] show typical features. They appeared as accidents both in adsorption isotherms and sound velocity curves. They are explained as being the onset of capillary condensation and the onset of pore filling. It is of a crucial importance to detect such effects when we deal with films uniformity which is still the subject of controversy in superfluid properties analysis $[3,4]$.

In this paper we present results obtained in the course of a study of third sound velocity in $\mathrm{He}^{3}-\mathrm{He}^{4}$ film mixtures reported elsewhere.

We report here experimental results of third sound velocity in $\mathrm{He}^{4}$ films coupled with isotherm adsorption measurements for different porous materials : alumina powder and papyex. Capillary condensation which is clearly observable, as is well known [1] by adsorption isotherms is also detectable with third sound. So this latter technique should be used with confidence as a film thickness probe when pressure measurements fail (at very low temperature for $\mathrm{He}^{4}$ films, or for $\mathrm{He}^{3}-\mathrm{He}^{4}$ mixtures).

Our cell contains a porous material $(P)$, and a glass substrate $(G)$ on which we measure third sound velocity. The porous material (alumina powder or papyex) has a very large surface adsorption area $A$, so that almost all of the introduced $\mathrm{He}^{4}$ gas is adsorbed on it. The adsorbed $\mathrm{He}^{4}$ volume $V$ is directly obtained from pressure variations in the room temperature calibrated reservoirs, a correction being made for the amount of helium which remains as vapor in the free volume of the cell.

We have first studied as porous material $2.3 \mathrm{~g}$ of $400 \AA$ alumina powder. The porosity of the powder (the ratio of open volume to total volume) is 0.92 ; its complete filling corresponds to $11.61 \mathrm{~cm}^{3}$ of $\mathrm{He}^{4}$. We have obtained at $2.2 \mathrm{~K}$ the classical isotherm by directly measuring the pressure $P$ in the cell with a digital pressure gauge [5]. The saturated vapour pressure $P_{0}$ at this temperature is measured by filling entirely the cell with $\mathrm{He}^{4}$. The adsorbed $\mathrm{He}^{4}$ volume $V$ is normalised by defining, as in ref. [1], the volume filling fraction $f=V / V_{\mathrm{t}} ; V_{\mathrm{t}}$ being the liquid volume filling entirely the porous material. The logarithmic representation (Fig. 1) of $f$ versus $\log P_{0} / P$ shows a

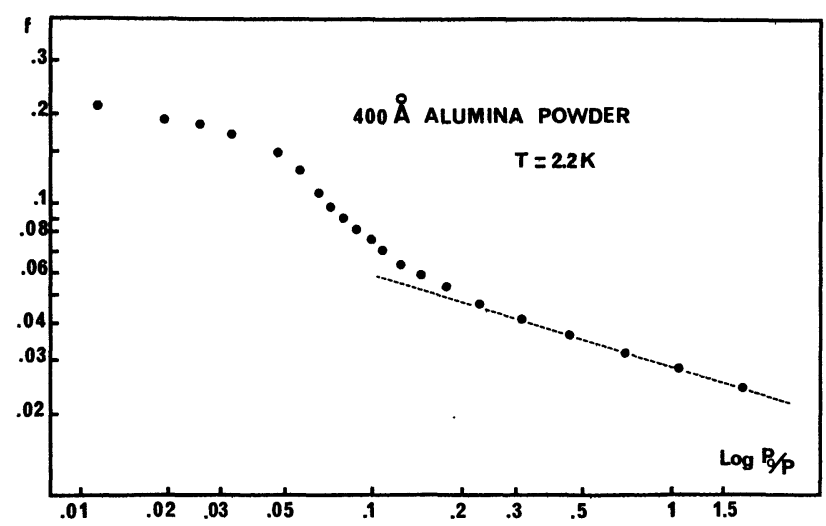

Fig. 1. - Classical adsorption isotherm on alumina powder. The capillary condensation onset is clearly visible at $\log P_{0} / P=0.23$. $P_{0}$ is the saturated vapour pressure of $\mathrm{He}$. 
pressure domain $\left(\log P_{0} / P \geqslant 0.23\right)$ where the slope is $-1 / 3$ (dashed line on figure 1 ). We thus experimentally find; as observed by other authors, that the Frenkel-Hasley-Hill (F.H.H.) description fits the adsorption isotherm in this pressure range. The deviation of such description occurs for $f=0.05$ and $\log P_{0} / P=0.23$ : as further liquid is added, the vapour pressure $P$ increases slowly with increasing fill. This behaviour has been convincingly identified in the literature $[1,6,7]$ as capillary condensation onset : added $\mathrm{He}^{4}$ atoms condensate at the contact points between the grains of powder, rather than increasing uniformly the thickness of the film.

We detect the capillary condensation onset for a value $\left(T \log P_{0} / P\right)^{-1 / 3}=1.25 \mathrm{~K}^{-1 / 3}$; this observation is in reasonnably good agreement with the value $\left(1.49 \mathrm{~K}^{-1 / 3}\right)$ obtained by Rosenbaum et al. [1] on $500 \AA$ alumina powder.

In addition to pressure measurements in the cell, we measure third sound velocity on the glass substrate (G), using, in the conventional way [8], two thin evaporated aluminium films respectively as heat pulse emitter and sensitive thermometer receiver.

As long as no capillary condensation occurs, the film thickness $d_{\mathrm{p}}$ on the porous material is given by :

$$
d_{\mathrm{p}}=\frac{V}{A} .
$$

When the F.H.H. description is valid [9], the film thickness $d_{\mathrm{G}}$ on the glass substrate is related to $d_{\mathrm{p}}$ within a good approximation by :

$$
d_{\mathrm{G}}=\left(\frac{\theta_{\mathrm{G}}}{\theta_{\mathrm{p}}}\right)^{1 / 3} d_{\mathrm{p}}
$$

where $\theta_{\mathbf{G}}$ and $\theta_{\mathbf{p}}$ are respectively the Van Der Waals interaction [10] constant between a $\mathrm{He}^{4}$ atom and glass or porous material. Then the usual relation between third sound velocity $C_{3}$ and film thickness $d_{\mathrm{G}}$ is [5] :

$$
C_{3}^{2} d_{\mathrm{G}}^{4}=B_{\mathrm{G}}\left(d_{\mathrm{G}}-D_{\mathrm{G}}\right)
$$

where $B_{\mathrm{G}}$ and $D_{\mathrm{G}}$ are constants. Using relations (1) and (2) we then obtain :

$$
C_{3}^{2} f^{4}=B_{\mathrm{P}}\left(f-f_{0}\right)
$$

with $f=V / V_{\mathrm{t}}$ as previously defined ; $f_{0}$ is a constant.

It is clear that a deviation of relation (1) implies a deviation of relation (4), which will also be a good indication of condensation capillary onset.

Our experimental results are presented figure 2 , where we have plotted at $1.4 \mathrm{~K}, C_{3}^{2} f^{4}$ versus $f$. They clearly show that the linear dependance of equation (4) fails for a filling fraction $f \simeq 0.05$, in good agreement with adsorption results of figure 1.

It should be noticed that the linear region (Fig. 2) is observed on a narrow range of the filling fraction $f$ : the low filling fraction range cannot be explored by third sound measurement as the $\mathrm{He}^{4}$ film is not yet superfluid. However it is important to notice that the intercept of the linear region (dashed line, figure 2)

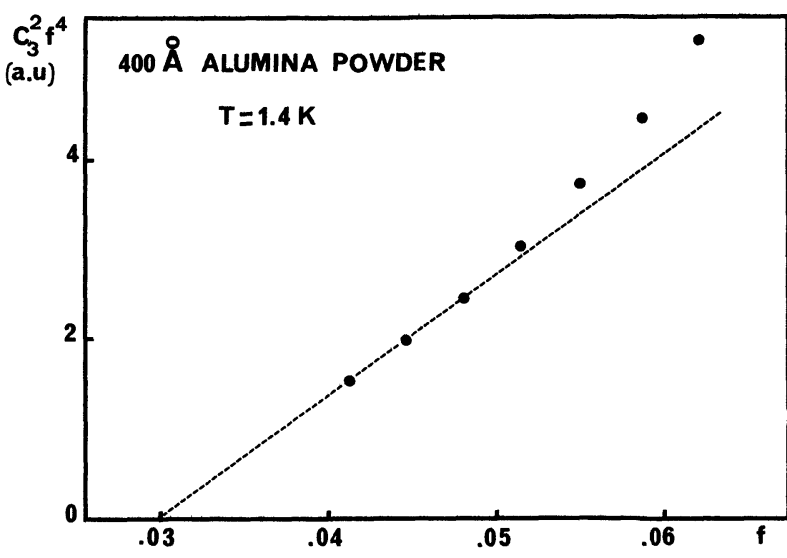

Fig. 2. - Representation of $C_{3}^{2} f^{4}$ versus the filling fraction $f$ for alumina powder. The departure of the experimental points from the linear variation predicted by eq. (4) indicates the onset of capillary condensation in the alumina powder. The intercept of the dashed line with the horizontal axis corresponds to a value of $D_{G}=2.2$ atomic layers, using $\theta_{\mathrm{G}}=27 \mathrm{~K}$ (at. layer) ${ }^{3}$.

with the horizontal axis, is $f=0.03$ which corresponds (using $\theta_{\mathrm{G}}=27 \mathrm{~K}$ (at. layer) ${ }^{3}$ ) to a film thickness on the glass substrate $D_{\mathrm{G}}=2.2$ atomic layers.

This intercept corresponds to the well known value $D$ of an $\mathrm{He}^{4}$ film thickness, for which the mean film superfluid density $\left\langle\rho_{\mathrm{s}}\right\rangle$ cancels. The value we find (2.2 atomic layers) is in very good agreement with results reported in the literature [8], which confirms the linear dependance of $C_{3}^{2} f^{4}$ before the capillary condensation.

We present in figure 3 the results obtained at $0.9 \mathrm{~K}$ on $11.4 \mathrm{~g}$ of papyex [11] with porosity 0.64 , and complete filling corresponds to $7.05 \mathrm{~cm}^{3}$ of $\mathrm{He}^{4}$. We observe capillary condensation, when the linear dependance fails [12], for a filling fraction $f=0.091$. This result was also confirmed by a classical isotherm at $2.2 \mathrm{~K}$ which indicates that capillary condensation occurs at this same filling fraction $f=0.091$ for $\log p_{0} / p=0.214$, that is to say for a value of

$$
\left(T \log P_{0} / P\right)^{-1 / 3}=1.29(\mathrm{~K})^{-1 / 3} \text {, }
$$

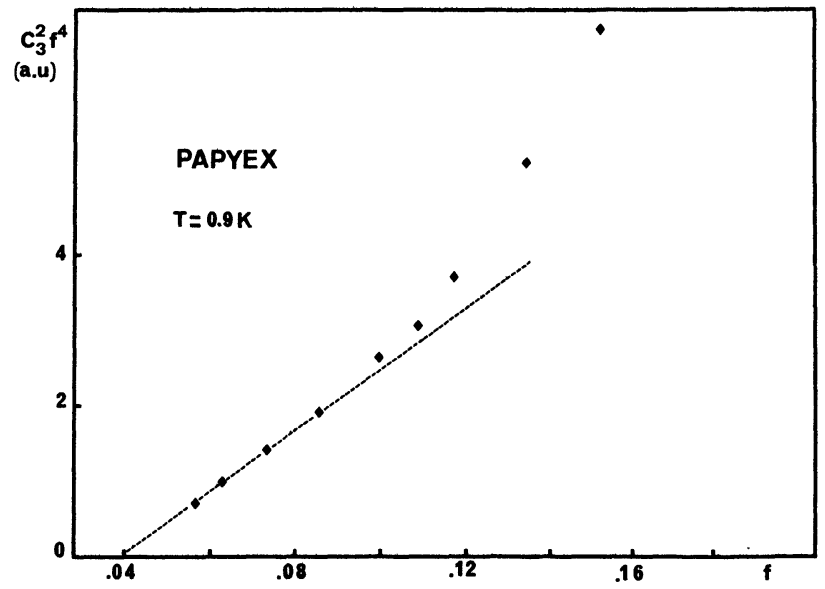

Fig. 3. - Representation of $C_{3}^{2} f^{4}$ versus filling fraction $f$ for papyex. Capillary condensation occurs for $f=0.09$. The intercept of the dashed line with the horizontal axis gives a value of $D_{\mathrm{G}}=1.66$ atomic layer, using $\theta_{G}=27 \mathrm{~K}$ (at. layer) ${ }^{3}$. 
greater than a rough estimate of the value obtained by Bienfait et al. [2] in graphite foam $\left(0.64(\mathrm{~K})^{-1 / 3}\right)$. This result indicates that capillary condensation would occur at lower uniform film thickness in graphite foam than on papyex which is compressed exfoliated graphite. However no conclusion can be drawn as the papyex we used was directly the commercial product.

Finally we mention that, from the classical isotherm, we can get the film thickness

$$
d_{\mathrm{G}}=\left(\frac{T}{\theta_{\mathrm{G}}} \log \frac{P_{0}}{P}\right)^{-1 / 3}
$$

on the glass substrate for a given filling fraction $f$.
With these computed values of $d_{\mathrm{G}}$ we have checked that relation (3) was correct well above the critical thickness at which capillary condensation occurs.

In conclusion this work shows that third sound velocity measurements are a good probe of film thickness which could be of a great help at very low temperature and for mixture films studies.

Acknowledgments. - One of us (J. P. L.) would like to thank Prof. Bienfait M., Dash J. G., Hallock R. B. and Reppy J. D. for many fruitfull discussions during the Phase transitions in surface films, Erice 1979 summer school, and Prof. Rudnick I. and Williams $\mathrm{G}$. for the very early discussions.

\section{References}

[1] Rosenbaum, R., Williams, G. A., Heckerman, D., Marcus, J., Scholler, D., Maynard, J. and Rudnick, I., to be published.

[2] Bienfait, M., Dash, J. G. and Stoltenberg, J., to be published.

[3] Dash, J. G., Phys. Rev. Lett. 41 (1978) 1178.

[4] Bishop, D. J. and Reppy, J. D., Phys. Rev. Lett. 40 (1978) 1727.

[5] MKS Baratron, Le Groupe Scientifique, La Plaine Saint Denis, France.

[6] Aristov, B. G., Karnaukhov, A. P., Kiselev, A. V., 'Russ. J. Phys. Chem. 34 (1960) 1019.

[7] In reference [1], Rosenbaum et al. have studied the same material, alumina powder, for different powder grain sizes. They found F.H.H. behaviour until onset capillary condensation occurs. They characterize onset capillary condensation both by adsorption isotherms and sound velocity measurements in the porous material.

[8] Scholtz, J. H., Mclean, E. D. and Rudnick, I., Phys. Rev. Lett. 32 (1974) 147.

[9] We are well aware that F.H.H. law is not generally valid as mentioned in reference [2]. However, in our experiments, we do observe F.H.H. behaviour before onset capillary condensation (see Fig. 1).

[10] Sabisky, E. S. and Anderson, C. H., Phys. Rev. A 7 (1973) 790.

[11] Papyex is the trade name of the product of Carbone Lorraine, 37-41 rue Jean-Jaurès, 92231 Gennevilliers (France).

[12] The intercept of the dashed line (Fig. 3) with the horizontal axis, gives us $D_{\mathrm{G}}=1.6$ atomic layers, again in very good agreement with results reported in the literature. 